\title{
Specific problems of FEM thermal simulations for microsystems
}

\author{
A. Götz, C. Cané, E. Lora-Tamayo \\ Centre Nacional de Microelectrònica (C.S.I.C.), Campus U.A.B., \\ E-08193 Bellaterra, Spain
}

\begin{abstract}
Microsystems often require special attention to the thermal aspects, as they frequently integrate severe heat sources together with electronics. When Finite Element Method programs are used for the solution of these problems, difficulties different from normal ICs are encountered, like, for example, thin membranes with huge aspect ratios and natural and forced convection in microcavities. Some of these aspects are discussed for the example of ANSYS 5.1 simulations of a 3-D multichip microsystem including a thermopneumatic micropump.
\end{abstract}

\section{Heat transport mechanisms in a microsystem}

Fig. 1 shows an example of a microsystem investigated in the European ESPRIT project BARMINT. It consists of a set of up to ten chips with variable functions. The chips are glued onto a silicon support, stacked and cast in epoxy. The resulting epoxy cube is reduced in size by sawing. During this step, the supports are also sawn through revealing cross-sections of the metal tracks. After metallizing the cube the metal is patterned by laser patterning. As well as the micropump the cube includes sensor, supply, test and signal processing chips. The sensor chip constitutes the bottom part of the liquid flow channel for the chemical and physical analysis and it is bonded onto a $1 \mathrm{~mm}$ thick PYREX glass. The system is designed as a demonstrator for compatibility for biomedical applications. The pump is driven by thermopneumatic actuation. The power management of the system is of prime importance: consumption of the pump should be minimized and the temperature at the chips containing electronics 


\section{Microsystems and Microstructures}

may not exceed the tolerable limits. Several technological solutions for the micropump are considered in the project. In the following discussion only a "hanging heater" design is considered, having the heating resistor placed on a membrane within the pump air chamber to provide good thermal isolation.

The possible heat transfer mechanisms are heat conduction, convection and radiation as summarized in Table 1 .

Table 1: Summary of heat transfer mechanisms.

\begin{tabular}{|c|c|c|c|}
\hline $\begin{array}{l}\text { Natural Convection } \\
\text { - Static fluid } \\
\text { - Laminar flow } \\
\text { - Turbulent flow }\end{array}$ & Conduction & $\underline{\text { Radiation }}$ & $\begin{array}{l}\text { Forced Convection } \\
\text { - Laminar flow } \\
\text { - Turbulent flow }\end{array}$ \\
\hline
\end{tabular}

In the case of the thermopneumatic pump the actuation principle is based on the thermal expansion of the air in the microcavity. The first point of interest is therefore the heat transfer to the gas. Firstly, simulations were done for studying natural convection, conduction and radiation. Simple samples were fabricated and measured and compared with the simulation. Afterwards, forced convection was also simulated and all the results were applied to the complete microsystem, finally calculating the membrane deflection as a function of heating power.

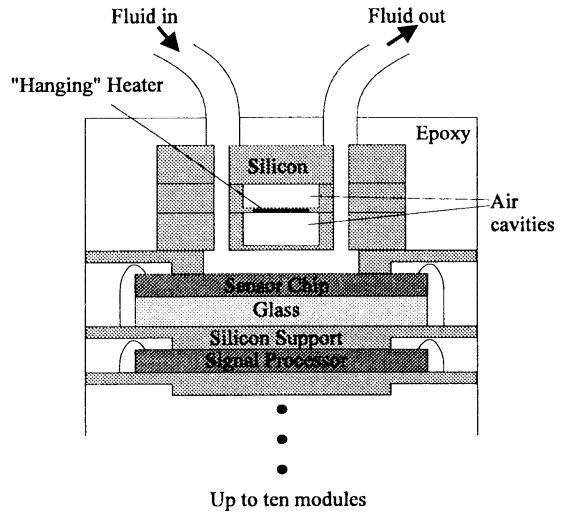

Figure 1: Microsystem including micropump.

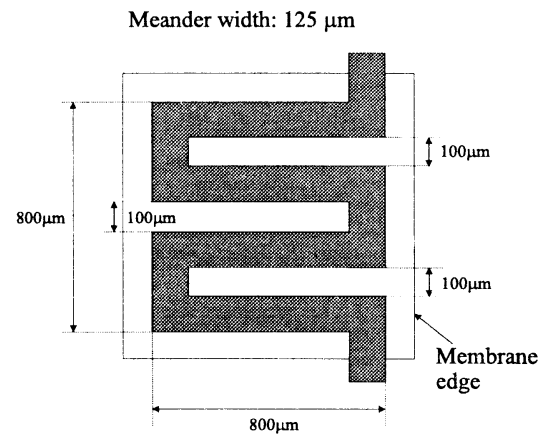

Figure 2: Heater layout of a measured sample.

\section{Samples and Measurements}

\section{Sample description}

The thermal behaviour of a set of simple samples was studied experimentally. The samples consisted of silicon chips with silicon nitride membranes etched 
anisotropically from the backside, supporting a meander shaped platinum heater (Fig.2 and Fig.4). The sample set included 8 samples with varying membrane dimensions and two different heater layouts. Membrane sizes and thicknesses were $1000 \times 1000$ or $1200 \times 1200 \mu^{2}$ and 100 or $150 \mathrm{~nm}$ respectively. The chip sizes were in the range of $5 \times 5 \mathrm{~mm}^{2}$. Heater layout \#1 is depicted in Fig.2. Heater layout \#2 is similar but the meander arm width is $100 \mu \mathrm{m}$ for the outer arms (horizontal and vertical) and $150 \mu \mathrm{m}$ for the two inner arms. Thickness of the Platinum heater was $30 \mathrm{~nm}$.

\section{The Measurements}

Measurements were performed in vacuum and in air in order to study conduction, radiation and especially to determine the influence of natural convection. The samples were heated and the resistance change and the power consumption of the heater were simultaneously recorded. The resistance change is a function of the average temperature change, as the temperature distribution is not uniform over the heater. Previously, the temperature coefficient of resistance of the platinum had been determined to be $\alpha=2.0 \times 10^{-3}$. Fig. 3 shows the measured power dissipation of a heated sample over the average temperature rise in vacuum and in air. The sample consisted of a $1200 \times 1200 \times 0.1 \mu \mathrm{m}^{3} \mathrm{Si}_{3} \mathrm{~N}_{4}$ membrane (heater layout \#1). As can be observed the most important heat path is the dissipation to the gas. As a consequence the first point studied was natural convection.

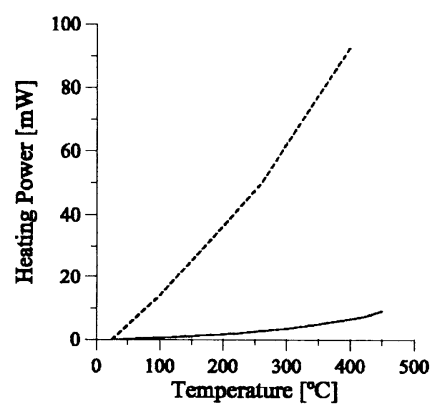

Figure 3: Measured power dissipation in air (dotted line) and vacuum.

\section{Natural (or free) convection}

Fluid motion can be caused by density differences and can be laminar or turbulent. When there is no fluid motion heat is transferred to the static gas by conduction. For the analysis of a convection problem it is essential to know the domain of flow. In the case of natural convection this is expressed by critical Rayleigh numbers for specific structures. In the case of a hot horizontal plate empirically found critical Rayleigh numbers are between 1100-1700 for the onset of laminar flow on top of the plate [1], about $1 \times 10^{5}$ for turbulent flow on top and $3 \times 10^{5}$ beneath the plate, and about $1 \times 10^{9}$ for vertical plates [2]. 
However, these critical Rayleigh numbers have been determined for macroscopic structures and it is doubtful if they can be used for the analysis of microscopic problems. For example Crary [1] uses them relating the characteristic length with the chip size. For a square chip of $1 \times 1 \mathrm{~cm}^{2}$ this gives critical temperature differences for the onset of laminar flow in the range of $10 \mathrm{~K}$. As the Rayleigh number depends on the third power of the characteristic length for smaller microsystems, Rayleigh numbers in the critical range are only computed for very high temperature differences.

\section{Simulation of natural convection}

ANSYS 5.1 provides a simple 4-node 2-D Thermal-Fluid element, FLUID15, which solves the flow equations along with the energy equation and is applicable to coupled thermal-fluid analysis. The fluid effects may be removed by completely defining the velocity degrees of freedom. The following assumptions are made: the fluid is incompressible, the flow is laminar, flow velocities are small so that the viscous dissipation can be neglected, and the fluid is supposed to be an ideal gas.

Simple axialsymmetric structures were modelled (see Fig.4). The choice of the flow domain is the responsibility of the analyst and can not be determined by the program. As the domain is unknown, all structures were simulated twice, once assuming static gas, solving only the heat conduction equation, and once assuming laminar flow, solving for both flow and temperature. If there is no significant change in the resulting temperatures the contribution of fluid motion to the total heat transfer can be neglected (although fluid motion may exist). Simulations were done using heat generation. The power-temperature relation was in the range of the measurement results. A variable in the simulations was cavity size produced by the package. Also, membrane and heater sizes were varied, while the chip size has little influence on the results. The surrounding air was modelled on top of and beneath the membrane. Fig.5 shows a typical resulting air flow. Membrane areas were simulated up to $1 \mathrm{~cm}^{2}$.

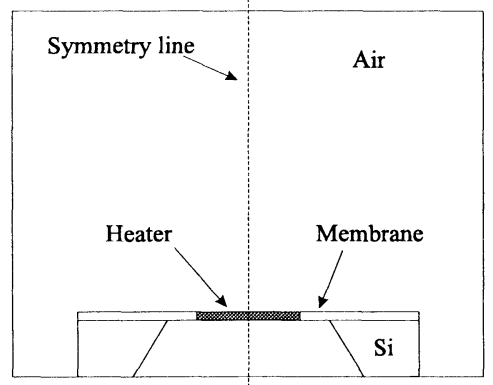

Figure 4: Modelled axialsymmetric structure.

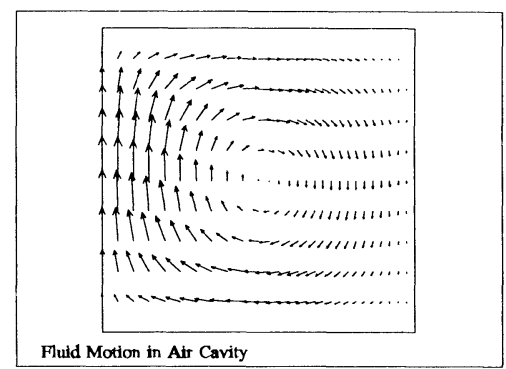

Figure 5: Typical flow on top of a heated membrane. 
Heating power was imposed to achieve temperatures up to $600^{\circ} \mathrm{C}$, as in some microsensor application (e.g. semiconductor gas sensors and pellistors) those temperatures are required. For microsystems with integrated electronics typical temperatures are much lower. The chips are supposed to be placed on a heat sink. A critical temperature is observed where the fluid starts to account for some heat transfer, depending on the size of the heated area, the isolation provided by the membrane and the cavity hight and side extensions. Fig. 6 shows a typical simulated power-temperature function disregarding fluid motion (linear dashed line) and considering it. Heater area and membrane diameter are $5 \mathrm{~mm}$ and $6 \mathrm{~mm}$ respectively. The $\mathrm{Si}_{3} \mathrm{~N}_{4}$ membrane is $200 \mathrm{~nm}$ thick and the air cavity on top $1 \mathrm{~cm}$ high and $2 \mathrm{~cm}$ in diameter. Flow velocities beneath the membrane were much smaller than on top of it and fluid motion in the bottom cavity could be neglected in all studied cases. The influence of fluid motion was found to be relatively small in all cases and was not considered in further simulations. This assumption is confirmed by the fact that the sample could be rotated at any angle and the dissipation did practically not vary. This was also observed by Lang [3]. In the case of the micropump presented, fluid motion inside the air cavity can be neglected due to the small cavity size and the relatively low temperatures.

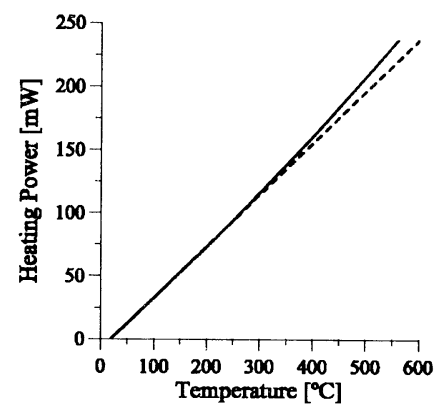

Figure 6: Heating power over maximum temperature considering fluid motion (continuous line) and disregarding it.

As fluid motion has no influence in the case of the microcavities studied, the problem reduces to a heat conduction problem. The heat transfer to the static gas due to conduction was consequently simulated.

\section{Heat Conduction}

\section{Modelling of Thin Membranes}

When the static gas is modelled with 3-D elements (SOLID70) it is imperative to model the membrane with 3-D elements also. The aspect ratio of the thin membranes, (ratio between lateral and vertical dimensions), is extremely high, and for the $30 \mathrm{~nm}$ thick platinum it is even worse. Modelling this structure with 3-D finite elements requires a very high number of elements. However, the 
membrane can be considered "thermally thin" (analogous to plane stress in mechanics) i.e. the temperature difference over the membrane thickness is so small (compared to the "interesting" temperature differences: the in-plane temperature distribution) that it can be assumed to be zero.

Although the membrane cannot be modelled with 2-D elements, simplifying assumptions can be made that reduce significantly the modelling effort:

- The multilayer structures can be reduced to monolayer structures.

When the thermal resistance of one layer is defined by:

$$
R_{t h, i}=\frac{1}{K_{i} \cdot t_{i}}
$$

where $K_{\mathrm{i}}$ and $\mathrm{t}_{\mathrm{i}}$ are the thermal conductivity and the thickness of one layer, then the total thermal resistance of the multilayer shunt gives:

$$
\frac{1}{R_{t h, t}}=\frac{1}{R_{t h, 1}}+\frac{1}{R_{t h, 2}}+\ldots . .+\frac{1}{R_{t h, n}}
$$

and

$$
K_{t} \cdot t_{t}=K_{1} \cdot t_{1}+K_{2} \cdot t_{2}+\ldots . .+K_{n} \cdot t_{n}
$$

- Buried layers can be modelled extended over the thickness of membrane.

The input power per volume has to be calculated with the new volume (new membrane thickness).

- The membrane can be modelled with one layer of elements only.

- Thin membranes can be substituted in the model by equivalent thicker ones. This can reduce significantly the total number of elements necessary. In this case, the in-plane thermal resistance has to be kept constant, i.e. when the thickness is increased by a certain factor the in-plane thermal conductivities have to by reduced by the same factor, while the conductivity in the plane normal direction has to be left unchanged. Defining different heat conductivities can be accomplished in ANSYS as it includes the possibility of orthotropic material properties.

The validity of the stated assumptions and approximations can be checked with simple models, where it is possible to mesh the actual 3-D structure and to compare the results with substitute equivalent structures.

\section{Heat conductivity of silicon nitride}

Heat conductivities for bulk silicon nitride are reported as high as $28 \mathrm{~W} / \mathrm{mK}$ [4] and as low as $2.25 \mathrm{~W} / \mathrm{mK}$ [3] for thin film PECVD $\mathrm{Si}_{3} \mathrm{~N}_{4}$. The value for the LPCVD $\mathrm{Si}_{3} \mathrm{~N}_{4}$ nitride membranes of the samples was therefore considered unknown. Measurements in vacuum at low temperature $\left(<150^{\circ} \mathrm{C}\right)$ were used to derive them. In this case heat conduction in the solid is the only transfer mechanism to be considered and the power dissipation over temperature behaviour is linear. The non-uniform power supply with the varying width of the heater arms and in the corners $\left(R_{c}=0.56 \times R_{\square}\right)$ is adjusted automatically when the heater shape is changed. As the measured temperature is an average temperature over the heater, this value is extracted from the simulation results 
with APDL (ANSYS Parametric Design Language). The heat conductivity was found to be $4.35 \mathrm{~W} / \mathrm{mK}$. Using this value for all samples, simulations matched the measurement within $3 \%$.

\section{Radiation}

Radiation was simulated using LINK31 elements on top and bottom of the membrane. In the case of vacuum measurements the measurement curve is traced very well for low temperatures $\left(<350^{\circ} \mathrm{C}\right)$ but for higher ones the losses are underestimated. This should be due to "hot spots" on the heater produced by the positive feed-back of locally supplied power and resistivity. On the other hand, measurements and simulations in air atmosphere showed that the most important heat path is conduction into the gas, while in this case radiation can be neglected.

\section{Comparison of Simulations and Measurements in Air}

The above results and assumptions were used for the simulations of the samples in air. To save elements the geometrical dimensions were reduced to a minimum by halving dimensions and comparing results. For air $0.5 \mathrm{~mm}$ on top is enough, as modelling a larger air volume does not bring about any further changes. The chip size can be reduced to $2 \times 2 \mathrm{~mm}^{2}$. Again, measurements and simulations were in very good agreement (mismatch $<5 \%$ ). Fig. 6 and Fig. 7 show the resulting heat distribution for heater type II and in comparison the observed glow behaviour of the Platinum heater.

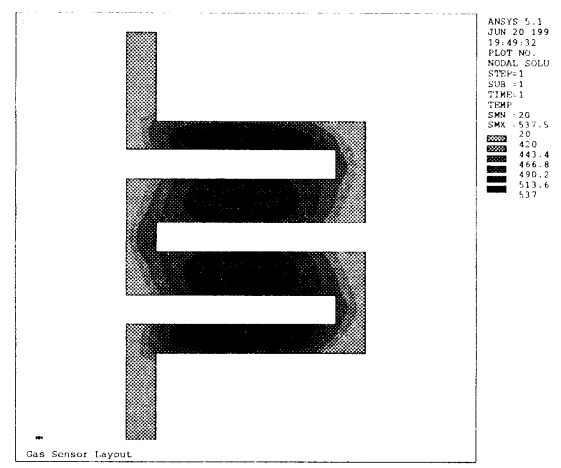

Figure 7: Simulated heat distribution over the heater.

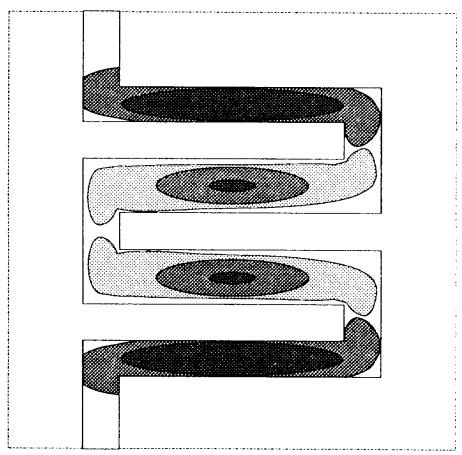

Figure 8: Optically observed glow behaviour of the platinium heater.

As in many applications a uniform temperature distribution is desired, other heater layouts were studied. As the temperature decreases towards the membrane edges, the first approach was a loop following the membrane edges. Very good results were achieved with a double loop configuration where the inner loop is 


\section{Microsystems and Microstructures}

thicker than the outer one. A similar heater design was designed and fabricated by M. Gall [5].

\section{Forced Convection}

The flux of liquid in the pump chamber of the micropump is a typical case of forced convection. The "heat transfer coefficient" is normally defined as a constant, related to a temperature, ("bulk temperature" in ANSYS and FLOTRAN), that can vary as a function of location, geometric dimensions, material parameters and flow. Alternatively, the bulk temperature can be defined constant and the heat transfer coefficient becomes a variable, which can be more convenient for the simulations. With the FLOTRAN program incorporated in ANSYS 5.1, forced convection was studied with the 2-D element FLUID142. For the heat transfer between fluid and solid the program provides the "preconditioned conjugate residual method". When the thermal material parameters of the fluid and the solid are very different, it is difficult to get a converged temperature solution. This is especially true when silicon is employed because of its high thermal conductivity. The simulated model was therefore as simple as possible. The liquid is assumed incompressible and the flow laminar, (small Reynolds number). The flow channel is continued before and after the membrane in order to avoid ill-conditioning by the definition of the inlet temperature of the fluid, set to ambient temperature. The solution could be brought to convergence using a relaxation factor of 1.0 for temperature and 30 search factors. Assumed flow was $1 \mathrm{ml} / \mathrm{min}$, the channel dimensions are: 300 $\mu \mathrm{m}$ high, $5.2 \mathrm{~mm}$ wide, and $10 \mathrm{~mm}$ long. Fig. 9 shows the heat transfer coefficient and the temperature at the interface. No measurement results are available at the moment, but the possibility to do measurements is being studied in the ongoing project.

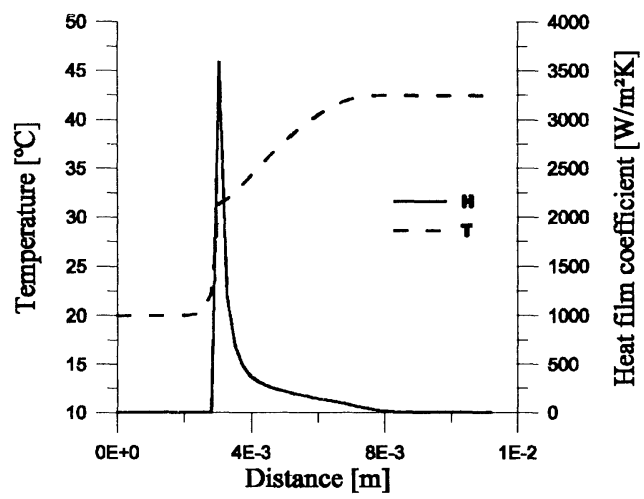

Figure 9: Heat transfer coefficient and temperature along the liquid cooled membrane. 


\section{2-D steady-state simulation of the microsystem}

PLANE55 2-D plane elements were used to get an approximate idea of the thermal behaviour of the complete microsystem. The heat transfer coefficient determined above is imposed on the membrane in contact with the liquid channel. As the inlet and outlet holes are small compared with the channel width, they are considered to have a small effect on the heat transfer. The resulting membrane deflection is computed by substituting the thermal elements by structural PLANE42 elements. The pressure produced in the cavity is computed by selecting the elements constituting the air in the pump cavity and calculating the average with APDL (The relative change of the volume is small compared to the relative pressure rise and is neglected). The air elements and thermal loads are removed before the structural analysis. Deformations of the structure by thermally induced stress in the solid parts can be taken into account by reading-in the calculated temperature distribution. The silicon is modelled using its orthotropic mechanical material constants [6]. Geometrical nonlinearities (large deflection and stress stiffening) are considered when the membrane deflections produced become relatively large $(>$ one fifth of membrane thickness). An example is summarized in Table 2.

Table 2: Thermal - mechanical steady-state behaviour of the micropump.

\begin{tabular}{|c|c|c|c|c|c|}
\hline $\begin{array}{c}\text { Heater } \\
\text { Membrane }\end{array}$ & $\begin{array}{c}\text { Pump } \\
\text { Membrane }\end{array}$ & $\begin{array}{c}\text { Constant } \\
\text { heating } \\
\text { power }\end{array}$ & $\begin{array}{c}\text { Average } \\
\text { T rise in } \\
\text { air cavity }\end{array}$ & $\begin{array}{c}\text { Average } \\
\text { P rise in } \\
\text { air cavity }\end{array}$ & $\begin{array}{c}\text { Max. } \\
\text { deflection }\end{array}$ \\
\hline $1 \mu \mathrm{miO}_{2}$ & $\begin{array}{c}20 \mu \mathrm{m} \\
\text { silicon }\end{array}$ & $10 \mathrm{~mW}$ & $9.8 \mathrm{~K}$ & $34 \mathrm{mbar}$ & $26.2 \mu \mathrm{m}$ \\
\hline
\end{tabular}

The membrane deflection is overestimated in the 2-D model. Therefore the pressure obtained was applied to a separate 3-D model of a perfectly clamped membrane and the results were compared. For the above case the 3-D model gives $19.9 \mu \mathrm{m}$ maximum deflection.

\section{Conclusions}

Some problems of thermal simulations of a microsystem were discussed. A set of assumptions used was validated with the help of measurements of simple samples. The results were then applied to the complete system.

\section{Acknowledgements}

The samples were prepared and measured at Deutsche Aerospace (Germany). Some of the simulations were done at Schlumberger Industry Research Centre (France) by the first author, during his diploma project [7]. Supervisors then 
146 Microsystems and Microstructures

were Prof. Dr. H. Ryssel from Fraunhofer IIS Erlangen (Germany) and Dr. J. Denis (Schlumberger, France).

\section{References}

1. Crary, S.B. Thermal Management of Integrated Microsensors, Sensors and Actuators, 1987, 12, 303-312.

2. Le Goff, Y. Transferts Thermiques, Lecture Notes, Ecole Nationale Superieure d'Arts et Metiers (ENSAM), Chalons-sur-Marne (France), 1991.

3. Lang, W. Heat Transport from a Chip, IEEE Transactions on Electron Devices, 1990, Vol.37, No.4, 958-963.

4. Milek, J.T. Silicon Nitride for Microelectronic Applications, Part 1: Preparation and Properties, IFI Plenum, New York, 1971.

5. Gall, M. The Si Planar Pellistor: a Low-power Pellistor Sensor in Si Thinfilm Technology, Sensors and Actuators B4, 553-538, 1991.

6. Wortman, J.J. \& Evans, R.A. Young's Modulus, Shear Modulus, and Poisson's Ratio in Silicon and Germanium, Journal of Applied Physics, 1965, Vol.16, No.1.

7. Götz, A. Optimierung der Temperaturverteilung und des Energieverbrauchs bei chemischen Sensoren, Diploma project, University of Erlangen-Nürnberg, Germany, 1993. 